\title{
AMÉLIORATION DE LA GESTION TECHNIQUE DES SALMONICULTURES PAR PROGRAMMATION SUR MICRO-ORDINATEUR
}

\author{
A. BELAUD, R. LABAT, P. LIM
}

Laboratoire d'Ichtyologie appliquée,

Ecole Nationale Supérieure Agronomique de Toulouse,

145. avenue de Muret, 31076 TOULOUSE Cédex, France

\section{RESUME}

Considérée comme trop complexe dans la pratique, la gestion technique des piscicultures a souvent été simplifiée, voire négligée.

Avec la prolifération des micro-ordinateurs, l'heure est venue de développer des logiciels de gestion de salmoniculture qui apporteraient, quotidiennement et sans difficulté, le service des connaissances scientifiques dans de domaine.

Cet article est une transcription en langage informatique des opérations de gestion technique de la pisciculture définies et parfois pratiquées antérieurement. Son originalité réside dans l'élaboration et l'utilisation d'une courbe de croissance ajustée aux performances réelles de la pisciculture étudiée.

Dans sa version actuelle, le programme donne les dates des divers stades de développement d'un lot de truites et, inversement, permet de déterminer la date de mise en incubation pour aboutir à une production de poids donné et à une date désirée.

Le programme permet surtout, pour chaque mois de l'élevage, d'établir une fiche contenant les bilans d'oxygène, d'excrétion d'ammoniaque et d'alimentation, ce qui définit le débit d'eau nécessaire et la masse des rejets polluants imputables à la pisciculture.

Ce programme, encore expérimental, nécessite d'autres extensions qui sont à l'étude et demandera ensuite d'être éprouvé aux réalités quotidiennes de l'exploitation piscicole.

\section{IMPROVEMENT OF TECHNICAL MANAGEMENT OF TROUT CULTURE BY MICROCOMPUTER PROGRAMMATION}

\section{SUMMARY :}

Considered in practice as too complex, the technical management of fish farms has often been simplified, even neglected.

With the proliferation of microcomputers it is time to elaborate specific management programs. These routines every day will bring to the fishfarmers the advantages of scientific knowledge in this field.

The present paper is a translation into an informatic language (APPLE's BASIC) of the series of the manual or graphic calculations previously used. Its originality lies in the elaboration of real growth curve of trout, fitted to the real performance of the studied farm.

In its actual version, the routine gives the dates of the principal development stages of a group of trout and reversely, the moment of hatching suitable to obtain a given production at a given date.

The program specially allows, month by month, to establish a report on oxygen, ammonia and feeding balances and consequently the water flow needed and the amount of wastes imputable to the farm.

The program. still at an experimental stage, needs further extensions which are under study and will afterwards be proved under the daily realities of fish farming.

\section{INTRODUCTION}

Une production piscicole performante est obligatoirement basée sur une croissance rapide des poissons, dans le double but de réduire le risque de maladies et de faciliter la rotation des bassins. Pour tendre vers cela, les poissons, de souche sélectionnée, doivent présenter le meilleur état physiologi- 
que, c'est-à-dire transformer le maximum de l'énergie des aliments pour que, les dépenses d'entretien couvertes, il puisse se dégager un bénéfice énergétique substantiel pour la synthèse de tissus vivants constituant la croissance. Pour réaliser ceci, le pisciculteur est, schématiquement, maître d'une part, de l'alimentation et, d'autre part, de l'eau apportant l'oxygène et emportant les déchets. Le problème est de rechercher la meilleure gestion possible de ces éléments de la vie des poissons pour tendre vers une productivité optimale.

Pour l'alimentation, les salmoniculteurs se basent le plus souvent sur des tableaux de nourrissage, indiqués pour chaque type d'aliment, à partir d'expériences d'optimisation de croissance réalisées en piscicultures-test. Certains pisciculteurs modulent ces indications en fonction de leurs propres observations sur la performance mais cette démarche reste basée sur des tâtonnements.

La gestion de l'eau est plus difficile du fait de la complexité des propriétés physico-chimiques, différentes d'un site à l'autre. II existe dans ce domaine des lois de dissolution des gaz et des lois d'équilibres chimiques mais d'application quotidienne complexe sans le secours d'un outil de calcul. En France, les bases de la gestion de l'eau ont été publiées dans diverses revues (FAURE. 1976: CEMAGREF, 1976 ; LELU, 1976).

Le développement de la micro-informatique éclaire sous un jour nouveau la gestion technique des piscicultures. Dorénavant, les calculs complexes peuvent être inclus dans des programmes et utilisés à volonté. II n'y aura dès lors aucun obstacle à l'expansion des méthodes de gestion qui tendront à exploiter au maximum les potentialités de production d'un site. Ces méthodes seront essentiellement basées sur des calculs de bilan d'oxygène (facteur limitant dans la plupart des cas), de bilan d'excrétion azotée (facteur limitant dans certaines eaux basiques). Un programme sur microordinateur permettra aussi de faire des calculs de bilan alimentaire, pour mieux adapter le nourrissage aux besoins réels des poissons et éviter le gaspillage qui se double de l'inconvénient de polluer la rivière.

S'il existe déjà des travaux sur l'utilisation de l'informatique pour la gestion sanitaire de la salmoniculture (HOSKINS et coll., 1979: TUFFERY 1979), le présent article constitue, à notre connaissance, une des premières approches des perspectives d'utilisation d'un outil informatique en gestion de croissance. Il s'appuie sur les expériences déjà effectuées à l'étranger (SPARRE, 1976) mais pour faciliter l'articulation avec les méthodes traditionnelles, il demeure calqué sur ces dernières pour ce qui concerne la nature et l'ordre des opérations de calcul. Le présent travail s'attache à définir une gestion qui s'appuie sur les bases physico-chimiques et physiologiques disponibles et qui pourrait facilement être mise à jour au fur et à mesure de l'avancement des connaissances dans ce domaine.

\section{PRÉSENTATION DES BASES DE GESTION RETENUES}

\section{I.O. PRINCIPE}

Pour cette première approche, il a d'abord été recensé, dans les articles précédents, les contraintes de la gestion technique des salmonicultures : principalement, l'apport d'oxygène et l'évacuation des déchets azotés toxiques (les contraintes imputables aux matières en suspension pourraient aisément être ajoutées). Ces éléments permettent, connaissant les propriétés physicochimiques de l'eau entrante, de calculer le débit minimal nécessaire pour un bassin. Pour ce faire, il faut aussi disposer d'informations sur les effectifs et sur la croissance. Cette dernière sera calculée soit directement à partir des résultats de pesées soit, à défaut, par adaptation de courbes de croissancetype puisées dans les revues techniques. Par comparaison entre l'aliment distribué et la croissance observée, il est enfin possible d'approcher le taux de conversion et les pertes de substances alimentaires qui accentuent la pollution des eaux sortant de la pisciculture. Le bilan alimentaire pourra être décomposé pour les lipides, protéines,... afin de mieux juger de la valeur de l'aliment utilisé.

Ainsi défini, le présent travail est schématisé dans la figure no 1 . Il repose sur trois bilans

\section{- bilan d'oxygène :}

Oxygène consọmé <ou = Oxygène disponible

(Oxygène disponible = oxygène apporté par l'eau, à concurrence des quantités correspondant à un seuil critique défini en I.1.2.)

\section{- bilan d'azote ammoniacal :}

Azote sortant de la pisciculture = azote dans l'eau à l'entrée + azote excrété par les poissons.

- bilan d'aliments :

Aliment consommé = aliment distribué - aliment et fèces emportés dans l'eau. 


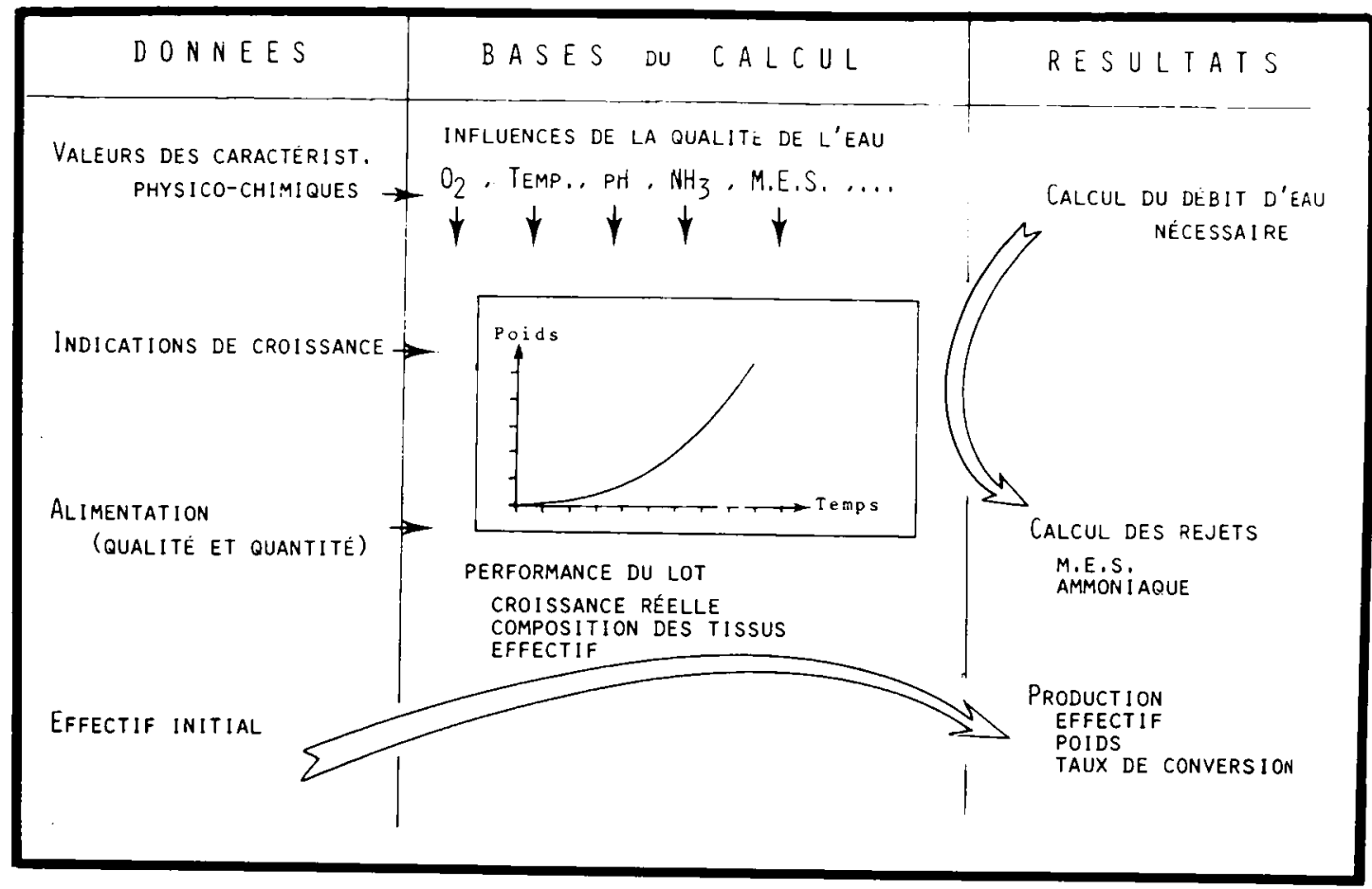

Figure 1 : La gestion nécessite la détermination des effectifs, des rations, de la croissance, de l'oxygène disponible et consommé, des capacités de l'eau dans l'évacuation de l'ammoniaque. Chacun de ces éléments est sous la dépendance de la température. En retour, le programme indiquera la quantité d'eau nécessaire, le taux de conversion et les pertes spécifiques de chaque constituant de la ration. Les propriétés physicochimiques de l'eau, la croissance et le rationnement étant définis une fois pour toutes pour chaque site, un calcul automatique donnera l'ensemble des résultats pour chaque lot de la pisciculture et pour chaque stade de développement des truites.

Figure 1: The technical management depends upon the number and size of trout, rate of feeding, growth, oxygen consumption and ammonia clearance. All of these characteristics are temperature-dependent. The program determines the minimal water flow, the food conversion index and the specific loss of each of the food constituents. The water properties, the usual growth performance of the farm and the feeding level are introduced as data. From these the routine performs, month by month, a report for each of the trout populations of the farm.

\section{I.1. LE BILAN D'OXYGENE}

\section{I.1.1. L'OXYGENE DANS L'EAU}

\section{I.1.1.1. Cas de la saturation à l'air.}

A l'équilibre avec l'air, l'eau né peut dissoudre qu'une quantité maximale d'oxygène, dont la valeur dépend de la température et de la composition ionique de l'eau. Pour une eau de salmoniculture, généralement peu minéralisée, on peut ne tenir compte que de l'effet de la température. Pour calculer les quantités d'oxygène dissous dans l'eau à saturation avec l'air, on peut appliquer diverses relations élaborées par les spécialistes. Le programme actuellement utilisé détermine la teneur en oxygène selon la formule de GREEN et CARRITT (1967)

Oxygène ( $\mathrm{mL} /$ litre sous $760 \mathrm{~mm}$ de mercure) $=-7.424+4.417 / \mathrm{T}-2.927 \times \log (\mathrm{T})+0.04238 \times \mathrm{T}$ (T étant la température absolue c'est-à-dire la température en degrés Celsius +273 ).

Dans le programme proposé, les valeurs sont corrigées d'une part pour tenir compte, à chaque température, de la valeur de tension de vapeur d'eau et, d'autre part, pour ajuster les valeurs en fonction de la pression atmosphérique moyenne calculée selon l'altitude du site. Pour la correction 
d'altitude, la relation: Pression moyenne $=760-0.0268638 \times$ Altitude (en mètres), a été introduite dans le programme. L'intérêt de l'ordinateur pour effectuer souvent ces calculs est évident.

Le calcul des valeurs de teneur en oxygène de l'eau pourrait sans difficulté être changé pour prendre en compte des travaux plus récents de BENSON et KRAUSE (1980) ou de MORTIMER (1982). Quelle que soit la relation utilisée pour définir la teneur en oxygène de l'eau, à saturation avec l'air ambiant, on peut convertir le résultat en $\mathrm{mg}$ /litre (ou p.p. m) en multipliant la valeur exprimée en $\mathrm{ml} /$ litre par le facteur $32 / 22.4$.

\subsubsection{Cas général}

En pisciculture, cet état de saturation par l'air ambiant ne constitue qu'un cas particulier et il faut connaître le pourcentage de saturation pour évaluer exactement la quantité d'oxygène renfermée dans l'eau. L'idéal est soit d'effectuer un dosage (méthode de WINKLER) qui donne directement le résultat, soit de procéder à des contrôles à l'aide d'un oxymètre qui indique le pourcentage de saturation par rapport à l'air. Cette connaissance des taux d'oxygène de l'eau entrante est déterminante et l'utilisation correcte d'oxymètres est à prescrire. A défaut d'introduire les vraies valeurs de concentration d'oxygène, le programme calcule les valeurs théoriques et gère sur cette base, aux risques et périls du pisciculteur qui ne procède pas aux contrôles ou qui utilise un oxymètre incorrectement étalonné.

\subsubsection{OXYGENE DISPONIBLE}

De par leurs caractéristiques physiologiques, les salmonidés sont exigeants en oxygène. Il est classiquement admis en pisciculture que les truites arc-en-ciel ne peuvent se développer correctement que si la concentration en oxygène de l'eau reste supérieure à 5 mg/I (ITAZAWA, 1971). Cette valeur d'oxygénation critique a le mérite de fixer les idées mais en réalité elle est arbitraire car susceptible d'être influencée par la température. la saison, la ration alimentaire, la composition de l'eau, la charge des bassins,... Des truites arc-en-ciel peuvent, par exemple, survivre plus de 24 heures à moins de $3 \mathrm{mg} / \mathrm{I}$ mais à jeûn et au repos dans des conditions d'expérimentation physiologique en laboratoire (THOMAS et HUGHES, 1982). II n'est certes pas souhaitable d'atteindre ces limites sublétales en pisciculture mais les recherches futures et les contrôles des taux d'oxygène effectués in situ devraient permettre de mieux cerner les valeurs de la limite d'oxygénation.

\section{I.1.3. CONSOMMATION D'OXYGENE DES POISSONS}

Bien qu'ayant fait l'objet de nombreuses études en laboratoire (WINBERG, 1960 ; HOCHACHKA et SOMERO, 1971 ; BRETT, $1976 ;.$.$) . la consommation d'oxygène des poissons est mal connue dans$ les conditions de pisciculture. Ceci s'explique par les difficultés de réaliser un bilan d'oxygène sur un bassin soumis à des échanges de gaz avec l'atmosphère. Il n'est cependant pas envisageable de transposer les résultats obtenus en laboratoire sur des poissons le plus souvent à jeûn et maintenus dans des volumes restreints. Ces expérimentations fondamentales indiquent comment la consommation d'oxygène des poissons est sous la dépendance de facteurs internes (espèce, taille, âge, sexe, stade de maturation gon adique, activité motrice, mode d'alimentation) et de facteurs externes (température, disponibilité de l'oxygène dans l'eau, composition de l'eau en ions, teneurs en azote ammoniacal, en dioxyde de carbone...), et ces données devraient être intégrées dans la gestion de l'eau en pisciculture.

En pratique, des pointages effectués dans plusieurs centaines de piscicultures aux Etats-Unis ont permis à LIAO (1971) de cerner la consommation d'oxygène des truites en élevage, en fonction des deux principaux facteurs que sont la température et le poids des poissons. Faute de données plus précises et dans l'attente de résultats qui relieraient la consommation d'oxygène des truites, mesurée en pisciculture, à un plus grand nombre de facteurs susceptibles d'influer, le programme de gestion proposé se base sur des seules données de LIAO.

Ces tables se traduisent par une relation logarithmique de la forme $\log \left(\mathrm{Mo}_{2}\right)=A \times \log$ (Poids) $+B$ avec $A \stackrel{2}{=} 0.8585$ et $B$, fonction de la température - aux températures inférieures à $11^{\circ} \mathrm{C}: \mathrm{B}=0.0575346 \times \mathrm{T}-0.966702$;

- aux températures supérieures à $11^{\circ} \mathrm{C}: \mathrm{B}=0.0239291 \times \mathrm{T}-0.593525$.

(le poids étant exprimé en grammes, $\mathrm{Mo}_{2}$ est obtenu en $\mathrm{mg} / \mathrm{kg} / \mathrm{heure}$ ). 


\section{I.2. BILAN DE MATIERES AZOTEES}

\subsubsection{BILAN GENERAL}

Pour faire le bilan des matières azotées, il faut d'abord connaître la quantité apportée avec l'aliment distribué (calculable si on relève, d'une part, la ration et, d'autre part, la composition de l'aliment indiquée par le fournisseur). Il faut ensuite évaluer, le cas échéant, la masse de matières azotées apportées par l'eau à l'entrée de la pisciculture. A la sortie d'un bassin, on doit retrouver les matières azotées de l'entrée, additionnées des matières apportées par l'aliment, excepté celles qui ont servi de matériel constitutif pour la croissance. Dans le calcul proposé dans le présent article. la croissance sera posée comme connue (Cf. ci-dessous) et il suffira de considérer que les tissus frais de truite renferment environ $14 \%$ de protéines (BORGSTROM, 1961 ; LOVE, 1980) pour estimer la fraction de matières azotées qui a servi pour synthétiser les nouveaux tissus. Compte tenu de la variabilité de la teneur en protéines des tissus de truites et donc de l'imprécision des valeurs moyennes utilisées, les molécules azotées autres que les protéines (acides nucléiques,...) pourront être négligées dans le cadre de ce travail. Le bilan se résume à :

Azote en sortie $=$ azote en entrée + azote de l'aliment - azote incorporé dans les tissus.

\subsubsection{BILAN D'AZOTE AMMONIACAL}

Parmi les substances azotées les formes ammoniacales revêtent un intérêt particulier, d'abord parce qu'elles constituent la majeure partie de l'excrétion azotée des poissons et ensuite en raison des effets toxiques qu'elles peuvent exercer.

\section{I.2.2.1. L'excrétion azotée}

On peut considérer que la quasi totalité de l'azote ingérée qui n'a pas servi à la construction de nouveaux tissus est excrétée sous forme ammoniacale (Cf. PROSSER et BROWN, 1961). La quantité d'azote ammoniacal excrété par les truites en élevage dépend de la charge des bassins, de la ration distribuée et aussi des facteurs qui influencent le métabolisme (essentiellement la température, l'espèce, la taille, l'âge...). Les pointages effectués en pisciculture par SPEECE (1973) conduisent à la relation d'estimation de la production d'azote ammoniacal suivante :

$M_{\mathrm{N} 2}=\exp ^{(\mathrm{B} \times \log (\mathrm{L}) \cdot \mathrm{C})}$

avec $B=-0.99$ et $C=0.126 \times T+0.42$ ( $T$ étant la température et $L$ désignant la taille en centimètres). Cette relation, établie en pisciculture, tient implicitement compte de l'auto-épuration bactérienne qui transforme une partie de l'azote ammoniacal excrété.

Pour homogénéiser cette relation avec celle du calcul de consommation d'oxygène, il est utile d'avoir une relation taille-poids valable pour les truites arc-en-ciel. Celle-ci a été obtenue par analyse des données du manuel technique SARB :

poids $(e n g)=0.0108067533 \times L^{3.01785179}$

ou longueur $(\mathrm{en} \mathrm{cm})=\exp ^{(\log (\mathrm{P}) \cdot \log (00108067533)) / 301785179}$

Le bilan d'azote ammoniacal s'établira ainsi

azote ammoniacal en sortie = azote ammoniacal en entrée + excrétion des poissons

En entrée de pisciculture, la teneur en azote ammoniacal n'est pas toujours négligeable en raison des apports de matières organiques en amont et du lessivage de sols amendés en azote. Dans ce cas un contrôle des concentrations de l'eau qui alimente la pisciculture s'impose. Ce contrôle serait renforcé dans les cas où le calcul de gestion technique désignerait l'ammoniaque comme facteur limitant de la charge du bassin.

\subsubsection{Toxicité de l'azote ammoniacal}

Dans l'eau, l'azote ammoniacal existe sous deux formes en équilibre : l'ion ammonium ( $\left.\mathrm{NH}_{4}^{+}\right)$, peu toxique et la forme non ionisée ( $\mathrm{NH}_{3}$ ou gaz ammoniac dissous), toxique pour des doses de l'ordre de $0.006 \mathrm{mg} / \mathrm{l}$ (SPEECE, 1973).

L'équilibre entre les deux formes s'écrit : $\mathrm{NH}_{3}+\mathrm{H}^{+} \rightleftharpoons \mathrm{NH}_{4}^{+}$

$L^{\prime}$ intervention de l'ion $\mathrm{H}^{+}$rend cet équilibre sensible à la valeur de $\mathrm{pH}$. Connaissant la valeur de $\mathrm{pH}$ et une valeur de constante d'équilibre (pK) donnée dans des tables, le rapport entre forme non ionisée et forme ionisée de l'azote ammoniacal se calcule selon l'équation :

$\mathrm{NH}_{3} / \mathrm{NH}_{4}=\exp (\mathrm{pH}-\mathrm{pK})$ 
Les valeurs de $\mathrm{pK}$ se situent entre 9.5 et 10 pour des températures comprises entre 14 et $6^{\circ} \mathrm{C}$. A titre d'exemple, pour une concentration en azote ammoniacal de $1 \mathrm{mg} / \mathrm{l}$, a $10^{\circ} \mathrm{C}$, il y aura $0.0058 \mathrm{mg} / \mathrm{I}$ d'ammoniac (donc pas de toxicité) à pH égal à 5.5, tandis que dans les mêmes conditions mais à $\mathrm{pH}=8$, le taux de $\mathrm{NH}_{3}$ atteindrait $0.18 \mathrm{mg} / \mathrm{l}$ et dépasserait ainsi largement le seuil de toxicité.

La gestion d'une pisciculture doit veiller à maintenir la concentration d'ammoniac en-dessous du seuil de $0.006 \mathrm{mg} / \mathrm{l}$ et cela nécessite une maîtrise du bilan d'ammoniaque doublée de contrôles fréquents des valeurs de $\mathrm{pH}$, surtout dans les eaux présentant un faible pouvoir tampon.

\section{I.3. BILAN ALIMENTAIRE}

\section{I.3.1. L'ALIMENTATION}

Le pisciculteur détermine l'apport quotidien d'aliment pour les truites de taille donnée et pour chaque valeur de température, selon les tableaux de rationnement mis au point par les fournisseurs et éprouvés en piscicultures-test. En plus de la ration conseillée, la composition de l'aliment est connue, du moins en ce qui concerne les teneurs en protéines, lipides, éléments minéraux et humidité. Il suffit alors de rentrer dans le programme de gestion le tableau de rationnement d'une part et les données qualitatives correspondant à l'aliment distribué, d'autre part. Dans son état actuel, le programme renferme les indications correspondant aux aliments "TROUW" et il pourrait être instantanément adapté à tout autre aliment du commerce.

L'intérêt d'une gestion technique assistée par ordinateur est de pouvoir mettre en parallèle l'aliment distribué et la croissance obtenue, pour juger de la valeur de l'aliment, évaluer les pertes et enfin pouvoir rectifier la quantité et la qualité de l'aliment pour tendre vers une efficacité optimale. L'ajustement de la formule de l'aliment paraît certes encore difficile à réaliser au niveau de la pisciculture mais il n'existe aucune difficulté de principe et il pourrait s'imposer dès lors que la rentabilité en serait démontrée.

\subsubsection{LA CROISSANCE}

Il est facile d'observer que, pour une alimentation donnée, la croissance est très variable selon la souche de truites, selon la technicité de la pisciculture et surtout selon les propriétés physicochimiques de l'eau. II n'était donc pas question, dans une gestion avancée, de se contenter de courbes de croissance-type définie pour l'ensemble des salmonicultures. Le présent article propose une méthode de détermination d'une courbe de croissance réellement valable pour la pisciculture étudiée.

Le point de départ idéal pour élaborer la courbe de croissance réelle serait de connaître, sur le plan fondamental, la part d’énergie métabolique réservée à la croissance. En réalité, les études physiologiques indiquent que l'enveloppe énergétique apportée par l'alimentation sert à couvrir d'abord les dépenses inévitables de routine (métabolisme de base, énergie nécessaire à la respiration. la digestion et à l'absorption des aliments, activité motrice de routine...) et ensuite les dépenses plus occasionnelles (activité motrice pour la recherche d'aliments ou d'oxygène...). C'est le bilan de ces dépenses qui déterminera s'il y aura ou non croissance selon que l'enveloppe énergétique est ou non excédentaire. La croissance est donc difficile à prévoir parce qu'elle résulte de nombreux éléments métaboliques et comportementaux et le problème se complique encore si l'on considère que chaque élément est influencé par les conditions d'environnement (température, propriétés chimiques de l'eau, type d'écoulement...). Enfin, ce bilan énergétique serait à reconsidérer pour chaque espèce et, au sein d'une espèce, pour chaque stade de développement. Les facteurs principaux restent cependant la température et le stade de développement (poids).

Devant la complexité du problème, on doit faire appel, ici encore, à des données empiriques obtenues par des relevés de croissance effectués sur le site même. Pour le pisciculteur qui procède régulièrement à des pesées, le problème est résolu et le programme de gestion se basera entièrement sur les indications injectées par l'utilisateur. Pour une utilisation plus générale du programme proposé, il fallait prévoir la possibilité d'établir une courbe de croissance complète à pártir des résultats partiels d'une ou plusieurs pesées et, à la limite, la possibilité de travailler sans aucune indication de pesée (courbe-type). II va de soi que les résultats correspondront d'autant mieux au cas de la pisciculture que le nombre d'indications de pesées sera élevé.

\section{I.3.2.1. Influence de la température}

Sur les conseils de pisciculteurs qui les avaient déjà testées, il a été retenu comme base de 
départ les valeurs d'accroissement de poids hebdomadaires de tables élaborées aux Etats-Unis par la firme SALMODYNE. Ces tables donnent des valeurs pour chaque température (entre 3 et $16^{\circ} \mathrm{C}$ ) et pour différentes valeurs de poids (de $0.2 \mathrm{~g}$ (démarrage) à $250 \mathrm{~g}$ ). 11 sera considéré que, en-dessous de $3^{\circ} \mathrm{C}$ et au-dessus de $16^{\circ} \mathrm{C}$. l'alimentation et donc la croissance est interrompue mais ceci poúrrait être revu pour adapter le programme à quelques cas particuliers.

L'analyse des valeurs d'accroissement montre que, à une température donnée, la croissance ne peut être décrite de manière satisfaisante par aucune fonction simple. II a donc été décidé de calculer la croissance point par point. A titre d'exemple et de référence, voici les poids obtenus chaque mois successif à $3^{\circ} \mathrm{C}$ et à partir d'un poids de démarrage de $0.2 \mathrm{~g}$ au mois zéro $: 0.378 ; 0.69 ; 1.21 ; 2.06 ;$ $3.39 ; 5.4 ; 8.4 ; 12.4 ; 17.8 ; 24.9 ; 33.6 ; 44.2 ; 56.6 ; 71 ; 86 ; 104: 122 ; 141 ; 161 ; 181 ; 202$; $225 \mathrm{~g}$ (cette courbe de croissance peut paraître optimiste en France mais ceci importe peu dans un travail qui ne retiendra de ces chiffres que l'allure générale de la courbe et où les indications issues de l'expérience de l'utilisateur recaleront les valeurs du poids moyen à chaque mois de développement).

Ces points de croissance théoriques, représentés dans la figure no 2 , correspondent à la température minimale pour la croissance, et, partant de là, il était nécesaire de construire la courbe correspondant à n'importe quelle autre température $t$.

Pour" modéliser "l'influence de la température, il a été fait appel à la notion de Q10. On définit classiquement ce facteur comme le rapport entre la valeur d'un processus (ici la croissance) à la température $\mathrm{t}+10^{\circ} \mathrm{C}$ et la valeur du même processus à la température $t$. Pour le cas où on comparerait la croissance entre deux températures $\mathrm{t} 1$ et $\mathrm{t} 2$ qui ne seraient pas exactement espacées de $10^{\circ} \mathrm{C}, 1 \mathrm{a}$ formule générale de calcul de Q10 serait :

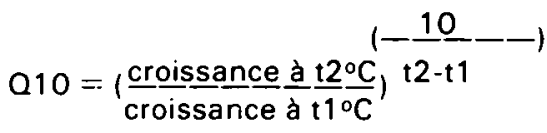

II restait à définir, pour chaque intervalle de températures situé entre la référence de $3^{\circ} \mathrm{C}$ et une température réelle $\mathrm{t}$, la valeur de $\mathrm{Q} 10$ qui, à partir de la croissance à $3^{\circ} \mathrm{C}$, permettrait de calculer la croissance à to $\mathrm{C}$. Pour ces valeurs de $\mathrm{Q} 10$, les relations obtenues grâce aux tables SALMODYNE sont les suivantes:

- entre 3 et $6^{\circ} \mathrm{C}$ :

- entre 6 et $8^{\circ} \mathrm{C}$ :

$\mathrm{Q} 10=0.064903 \times \mathrm{P}+0.868$

$\mathrm{Q} 10=0.025354 \times \mathrm{P}+1.491$

- entre 8 et $10^{\circ} \mathrm{C}: \quad \mathrm{Q} 10=0.015219 \times \mathrm{P}+1.576$

- entre 10 et $12^{\circ} \mathrm{C}: \mathrm{Q} 10=0.010411 \times \mathrm{P}+1.595$;

- entre 12 et $14^{\circ} \mathrm{C}: \mathrm{Q} 10=0.007577 \times \mathrm{P}+1.609$;

- entre 14 et $16^{\circ} \mathrm{C}: \mathrm{Q} 10=0.005287 \times \mathrm{P}+1.748$.

¿P désigne le poids en grammes).

Les points de croissance de référence à $3^{\circ} \mathrm{C}$ permettent donc de calculer les points de croissance à n'importe quelle autre température tà condition d'utiliser le facteur Q10 valable pour la gamme thermique et pour le poids des truites. Ces points demeurent cependant ceux d'une courbetype et il est nécessaire de les rectifier pour tenir compte des conditions réelles de la pisciculture. définies par des résultats de pesée(s)

\subsubsection{Rectification des points des courbes-type.}

Cette partie s'adresse aux exploitants qui possèdent des relevés de croissance : la qualité des résultats sera fonction du nombre et de la précision de ces relevés. A défaut, les calculs s'effectueraient sur la base des courbes-type définies ci-dessus.

Le programme procède dans l'ordre suivant (figure n०2):

- il prend les points de référence de la courbe-type définie plus haut et à $3^{\circ} \mathrm{C}$;

- il calcule les points correspondants à la température de la pisciculture $\mathrm{t}^{\circ} \mathrm{C}$ selon la méthode présentée ci-dessus (utilisation du Q10):

- il relève le point de cette dernière courbe correspondant à l'àge des poissons qui ont été pesés (soit Pref. ce poids théorique de référence) :

- il compare la valeur du poids réel obtenu à la pesée (P) à celle de Pref. :

- il rectifie tous les points de la courbe a la température $t^{\circ} \mathrm{C}$ en les affectant du coefficient $\mathrm{P} / \mathrm{Pref}$. :

- il obtient ainsi les points d'une croissance rectifiée, pour la température $t^{\circ} \mathrm{C}$;

- il calcule, à partir des points précédents, les points qui correspondent à $3^{\circ} \mathrm{C}$ (par utilisation du $Q 10$ correspondant) et ces points vont servir de nouvelle référence, adaptée à la pisciculture, pour les calculs ultérieurs. 


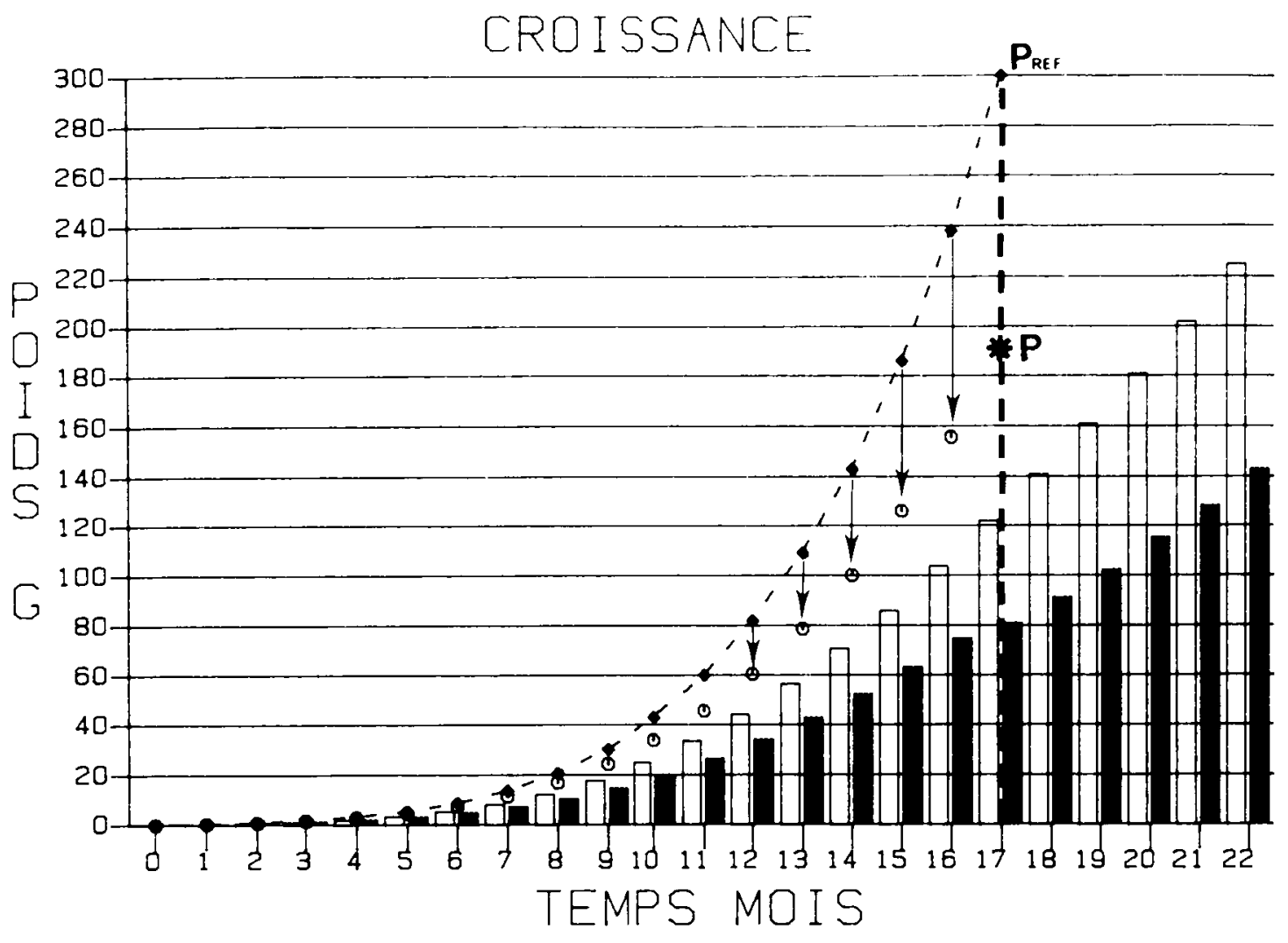

Figure 2: Elaboration d'une courbe de croissance adaptée à la pisciculture étudiée, à partir $d^{\prime}$ une courbe-type de croissance à $3^{\circ} \mathrm{C}$ (représentée par des barres ouvertes) et à partir d'un relevé de pesée effectuée dans la pisciculture étudiée (truites de $190 \mathrm{~g}$ à 17 mois et à la température moyenne de $13^{\circ} \mathrm{C}$. La courbe-type à $3^{\circ} \mathrm{C}$ permet d'obtenir la courbe-type à $13^{\circ} \mathrm{C}$ (en pointillés) par application d'une loi de $Q 10$. En rectifiant cette dernière courbe selon le coefficient $P$ / Pref (poids réel à la pesée/poids prévisible en se basant sur la courbe-type), on obtient tous les points de la croissance à $13^{\circ} \mathrm{C}$ (cercles ouverts). En repassant a $3^{\circ} \mathrm{C}$ par une loi de 010 , on élabore une courbe de référence adaptée a la pisciculture (barres pleines), et disponible pour les calculs ultérieurs.

Figure 2 : Working out, from a standard growth curve corresponding to a $3^{\circ} \mathrm{C}$ temperature (open bars), of the growth curve specific to the studied fish farm by using a set of weighing data (i.e. trouts of $190 \mathrm{~g}$ average weight, aged 17 months, at an average temperature of $13^{\circ} \mathrm{C}$. The standard curve at $3^{\circ} \mathrm{C}$ allows to obtain the type-curve at $13^{\circ} \mathrm{C}$ (dashed line) according to a $Q 10$ relation). This last line is rectified point by point, using the coefficient $P /$ Pref (real weight/theorical weight predicted with the dashed type-curve $13^{\circ} \mathrm{C}$ ). leading to the real growth curve at $13^{\circ} \mathrm{C}$ (open circles). By means of the 010 relation, this last real growth curve at $13^{\circ} \mathrm{C}$ is converted to a definitive reference growth curve at $3^{\circ} \mathrm{C}$ (filled bars) specific of the present farm and which will be available for further calculations.

Ces opérations peuvent être réalisées dès lors que l'on introduit un seul relevé de pesée incluant la température. le poids et l'âge des truites. Si l'on ne dispose que d'un seul relevé, tous les points de la courbe-type sont rectifiés sur la base de ce seul pointage. Dans l'hypothèse où l'on introduir ait plusieurs relevés correspondant à des phases de développement différentes. la rectification pourrait se faire de manière plus précise au sein de chaque intervalle de poids compris entre deux pesées.

\section{I.3.2.3. Utilisation des courbes de croissance rectifiées.}

L'avantage des points de croissance adaptés au cas de la pisciculture n'est pas seulement de tenir compte de l'expérience du pisciculteur, il réside aussi dans la possibilité de prévoir une crois- 
sance dans des conditions de température fluctuantes puisqu'on passe simplement d'une valeur de croissance de référence à $3^{\circ} \mathrm{C}$ à la valeur correspondant à une température quelconque en prenant la valeur de $\mathrm{Q} 10$ adéquate : rien n'interdit de changer la température chaque mois, à condition de relever la bonne valeur de $Q 10$. Dans sa version actuelle, le programme calcule mois par mois mais rien ne s'oppose fondamentalement à l'adapter au travail semaine par semaine et même jour par jour (si la taille des mémoir es de l'ordinateur le permet). Du moment que le programme, dans son état actuel, ne prend pas en compte les événements imprévisibles (maladies, anomalies climatiques,...), il ne paraît cependant pas utile d'affiner les calculs au-delà de la périodicité du mois. La figure $n^{\circ} 3$ donne un exemple de simulation de croissance en pisciculture où la température change chaque mois.

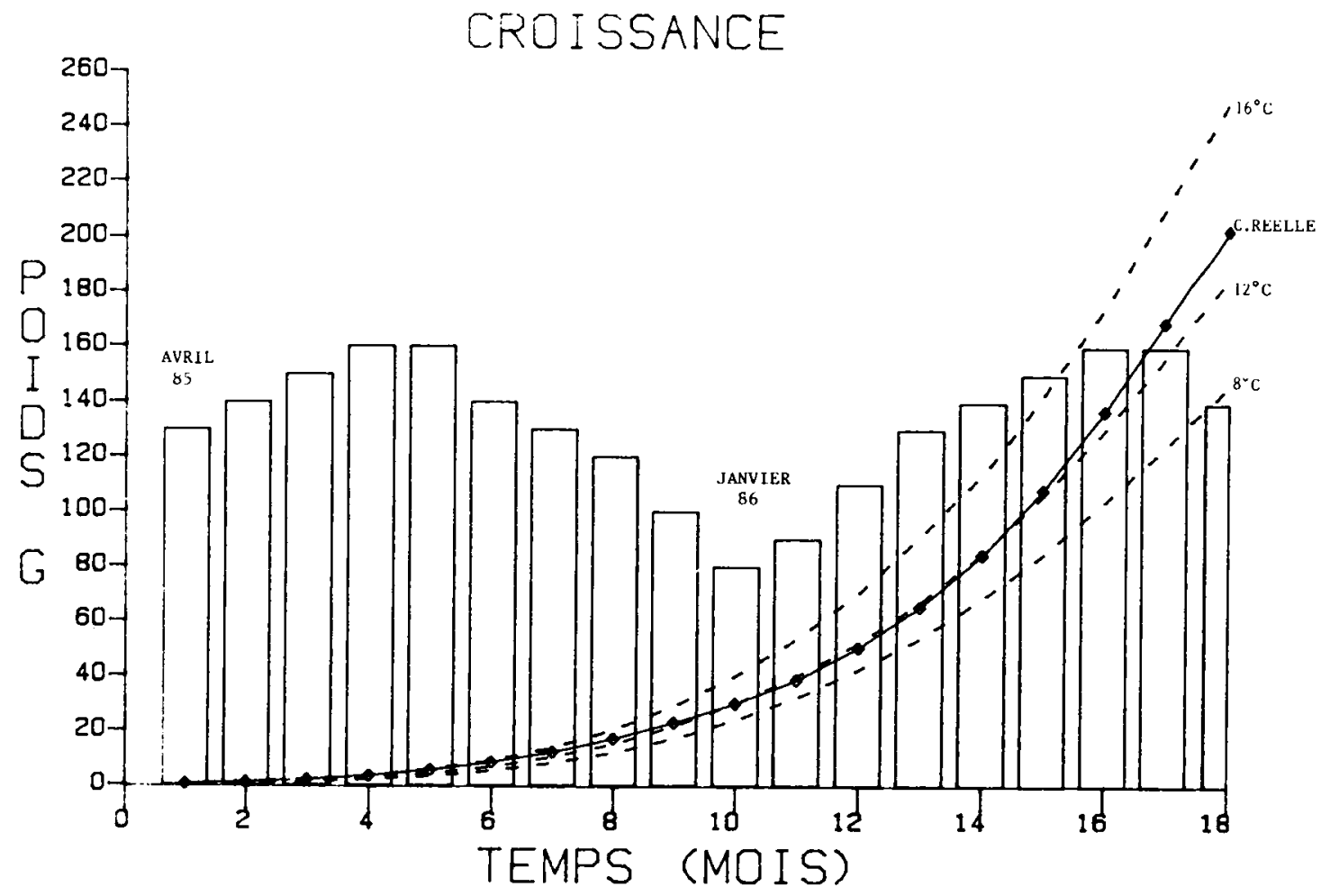

Figure 3: Exemple de courbe de croissance adaptée à une pisciculture (en trait plein) tenant compte de l'évolution annuelle des températures (les valeurs de température, figurées par des barres ouvertes, se lisent en divisant par dix l'échelle des poids). En pointillés sont représentées les courbes qui auraient été obtenues aux températures fixes de 8, 12 et $16^{\circ} \mathrm{C}$, respectivement.

La croissance réelle se situe tantôt en-dessus (l'été), tantôt en-dessous (I'hiver) de la courbe $12^{\circ} \mathrm{C}$ (cette dernière correspondant à la température moyenne de la pisciculture).

Figure 3 : An example of growth curve worked out for an hypothetical fish farm (full line) regarding the annual temperature variations (the values for temperature (indicated by open bars) are read in ${ }^{\circ} \mathrm{C}$ on the weight axe by dividing by 10 the corresponding value). The dashed lines are the isotherm growth curves calculated for 8,12 and $16{ }^{\circ} \mathrm{C}$ respectively.

Regarding the isotherm curve at $12^{\circ} \mathrm{C}$ (corresponding to the average of the annual temperature), the real growth curve (full line) is over (summer conditions) or under (winter).

\section{LE PROGRAMME (ETAT AU 1'r FEVRIER 1985)}

La programmation a été réalisée en langage BASIC sur micro-ordinateur APPLE II, de mémoire $128 \mathrm{k}$ octets, équipé d'un lecteur de disquettes et d'une imprimante. Le programme occupe un espace mémoire de $21 \mathrm{k}$ octets. 
Le programme contient les éléments de calcul exposés plus haut, chaque type de calcul étant isolé dans un sous-programme. Les données rarement appelées à être modifiées sont figées dans les lignes de programme (composition des tissus de truite, composition des aliments, points de la courbe de croissance-type,...) tandis que les données propres à chaque pisciculture sont demandées à l'écran et introduites par l'utilisateur. L'ensemble des données est classé en deux sous-ensembles (les caractéristiques physico-chimiques de l'eau d'une part, et les caractéristiques de la croissance. d'autre part) puis conservées dans des fichiers enregistrés sur le disque. Chaque programme de calcul appelle les données qui lui sont nécessaires

A la mise sous tension, l'écran affiche un menu qui offre les choix suivants

1 * Premier contact :

2 * Consultation des caractéristiques physico-chimiques:

3 * Consultation des caractéristiques de la croissance ;

4 * Impression des caractéristiques physico-chimiques ;

5 * Impression des caractéristiques de la croissance ;

6 * Correction des caractéristiques physico-chimiques ;

7 * Correction des caractéristiques de la croissance :

8 * Détermination des principales dates du développement des truites à partir de la mise en incubation ;

9 * Détermination des principales dates connaissant les dates et les tailles de commercialisation :

10 * Suivi d'un lot avec impression des valeurs calculées mois par mois

L'option 1 * contient, pour la première utilisation, un questionnaire obligatoire sur les propriétés de l'eau de la pisciculture au long d'un cycle annuel. Les valeurs à préciser sont pour chaque mois, le débit disponible, la température, l'oxygénation, le $\mathrm{pH}$, la concentration en ammoniac et en matières en suspension. A défaut de connaitre les valeurs mensuelles, le programme adopte des valeurs de base : 7 pour le $\mathrm{pH}, \mathrm{O}$ pour l'ammoniac et les matières en suspension et la valeur de saturation théorique pour l'oxygène. Le programme n'aurait aucune portée sans la connaissance des valeurs de débit et température mensuelle ; le nombre de données effectivement relevées par le pisciculteur détermine la qualité des calculs ultérieurs. A titre d'exemple, le tableau no 1 reproduit les valeurs des caractéristiques physico-chimiques de l'eau introduites en mémoire lors de ce premier contact.

L'option 1 * établit aussi les points d'une courbe de croissance adaptée au cas de la pisciculture selon la procédure exposée plus haut et illustrée par le graphique de la figure $n^{\circ} 2$. L'utilisateur doit se munir pour cela de relevés de pesées effectués dans la pisciculture même et la qualité de ces relevés est déterminante pour la suite de ce travail sur micro-ordinateur.

\begin{tabular}{|c|c|c|c|c|c|c|c|c|c|c|c|c|}
\hline $\begin{array}{l}\text { File: PROPRIETES DE L } \\
\text { Report: piscicul ture } \\
\text { CARACIERISTIQUE }\end{array}$ & JANVIER & FEVRIER & MARS & AVRIL & $\mathrm{MAI}$ & JUIN & JUILLET & AOUT & SEPTEMBRE & OCTOBRE & NOVEHBRE & DECEMBRE \\
\hline $\begin{array}{l}\text { Debit }(\bullet / \mathrm{H}) \\
\text { Teap.bassins }\left({ }^{\circ} \mathrm{C}\right) \\
\text { Tesp.incubation } \\
\text { Oxygene (ag/l) } \\
\text { pH (unité pH) } \\
\text { N-anoniacal (ng/l) } \\
\text { Katieres en suspension }\end{array}$ & $\begin{array}{l}1800 \\
8 \\
8 \\
11.54 \\
6 \\
0 \\
0\end{array}$ & $\begin{array}{l}2160 \\
9 \\
9 \\
11.27 \\
6 \\
0 \\
0\end{array}$ & $\begin{array}{l}2340 \\
11 \\
11 \\
10.76 \\
6 \\
0 \\
0\end{array}$ & $\begin{array}{l}1800 \\
13 \\
13 \\
10.29 \\
6 \\
0 \\
0\end{array}$ & $\begin{array}{l}1620 \\
14 \\
14 \\
10.06 \\
6 \\
0 \\
0\end{array}$ & $\begin{array}{l}1440 \\
15 \\
15 \\
9.85 \\
6 \\
0 \\
0\end{array}$ & $\begin{array}{l}1080 \\
16 \\
16 \\
9.65 \\
6 \\
0 \\
0\end{array}$ & $\begin{array}{l}900 \\
16 \\
16 \\
9.65 \\
6 \\
0 \\
0\end{array}$ & $\begin{array}{l}1080 \\
14 \\
14 \\
10.06 \\
6 \\
0 \\
0\end{array}$ & $\begin{array}{l}1440 \\
13 \\
13 \\
10.29 \\
6 \\
0 \\
0\end{array}$ & $\begin{array}{l}1800 \\
12 \\
12 \\
10.52 \\
6 \\
0 \\
0\end{array}$ & $\begin{array}{l}1980 \\
10 \\
10 \\
11 \\
6 \\
0 \\
0\end{array}$ \\
\hline
\end{tabular}

Tableau 1: Récapitulation des valeurs des caractéristiques physico-chimiques prises en compte à l'entrée de la pisciculture. Moyennes mensuelles.

Table 1: Values of the inlet water properties for each month of the year taken into account for the calculations.

Les options 2 * et $3^{*}$ permettent d'appeler et afficher à l'écran les valeurs, respectivement, des caractéristiques physico-chimiques et des caractéristiques de la croissance introduites et enregistrées lors d'une utilisation préalable en option " premier contact ".

Les options $4^{*}$ et 5 * assurent le même travail que les deux précédentes mais avec édition sur imprimante des données en vigueur.

Les options $6^{*}$ et 7 * permettent de modifier les données (respectivement les données de physico-chimie et celles de la croissance) puis de stocker les nouvelles valeurs sur disquette en place des précédentes qui ont été jugées erronnées. 
L'option 8 *, partant de la date de mise en incubation, détermine les dates d'éclosion, de démarrage et suit, mois par mois, l'évolution du poids moyen. Ces calculs tiennent compte des données physico-chimiques correspondant à chaque date et des performances de croissance de la pisciculture.

L'option 9 * effectue un calcul inverse du précédent : il remonte à la date de mise en incubation en partant de la date où l'on souhaite des truites de poids indiqué. Cet élément de programme planifie l'élevage en vue de réaliser une production définie par une date et un poids.

L'option 10 *, la plus substantielle, procède, mois d'élevage par mois d'élevage, à une prévision de suivi. Les résultats qui s'impriment comprennent l'effectif, les poids et taille moyens, les consommation d'oxygène et production d'azote ammoniacal individuelles et totales, la concentration d'ammoniac. Ces premiers résultats conduisent à déterminer le débit minimal et donc à la gestion de la masse d'eau nécessaire. L'impression se poursuit par la table de rationnement et le type d'aliment suggéré, puis par celle d'un bilan alimentaire où figure le taux de conversion pour l'ensemble de l'aliment puis pour chacun des principaux constituants de la ration, en particulier les protéines. Ce

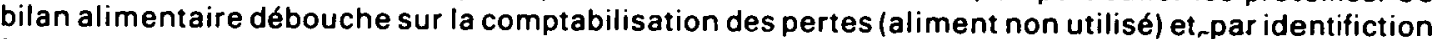
à ces dernières, à celui des matières polluantes (en suspension et dissoutes) introduites par la pisciculture. Le tableau $n^{\circ} 2$ présente un extrait de fiche de suivi valable pour un $8^{\circ}$ mois de croissance, dans une pisciculture fictive, volontairement choisie non performante pour mieux présenter l'étalement des courbes de croissance dans les figures de cet article.

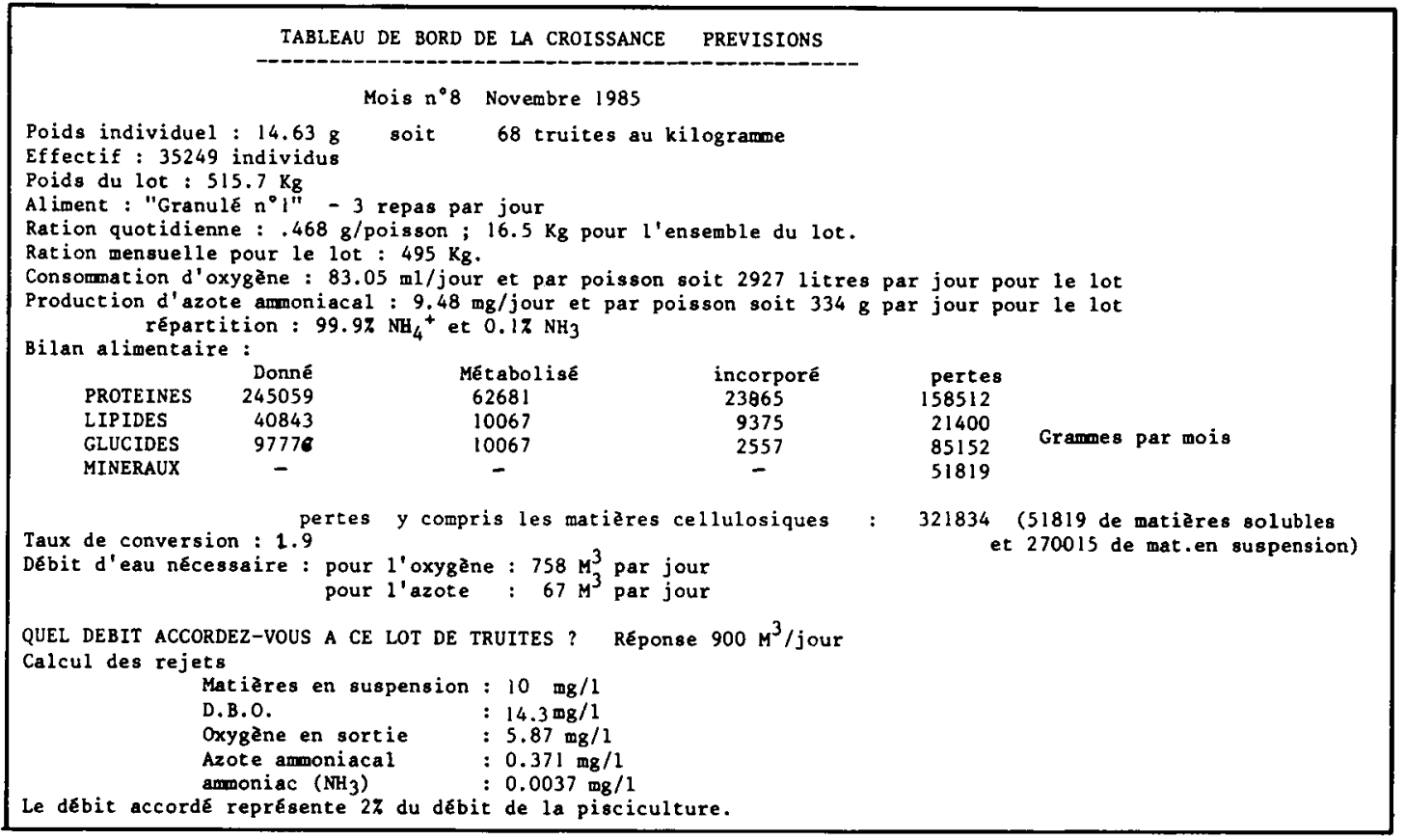

Tableau 2 : Exemple de résultat obtenu avec l'option du programme 10 * et se rapportant au huitième mois de croissance d'un lot hypothétique.

Table 2 : Example of a report obtained by using the 10 * routine and corresponding to a 8 months aged group of trout.

\section{PREMIER BILAN ET PERSPECTIVES}

Le principal intérêt de cette gestion assistée par micro-ordinateur est de conduire, en option 9 * à l'évaluation objective du bilan alimentaire. Connaissant la part de l'aliment dans les coûts de production, l'utilisateur doit, dès la première utilisation d'un tel programme, obtenir un résultat intéressant. Les premières applications font ressortir que, dans les piscicultures à croissance lente, il y a souvent sur-alimentation des poissons, au détriment du coût de production et de la préservation de l'environnement aquatique à l'aval. Une gestion améliorée doit conduire à réduire les taux de conver- 
sion dans beaucoup de piscicultures, sans doute au prix d'une formulation de l'aliment " personnalisée " pour chaque pisciculture

Les premières indications concernant l'utilisation des protéines semblent montrer que. si le bilan est souvent excédentaire pour les truites de plus de 10 grammes, il est limite et voire déficitaire au démarrage des truites. Si ces résultats étaient confirmés dans le cadre d'une utilisation intensive de ce type de programme, il conviendrait de se tourner vers les fabricants d'aliments pour suggérer un réajustement des formules. De toute manière ce programme fait toucher du doigt - pour ceux qui resteraient à convaincre - que chaque pisciculture devrait avoir sa propre formulation, établie en relation avec sa propre performance de croissance. Même si ceci paraît difficile à envisager dans le contexte actuel, l'avenir pourrait imposer l'idée d'un aliment composé " à la carte ".

Pour ce qui est de la détermination des dates des principales phases d'élevage, elle est généralement bien maîtrisée par les pisciculteurs expérimentés. Le programme proposé facilite ce travail de prévision et le rend plus fiable d'une part en le fondant sur la croissance réellement observée dans la pisciculture en question et, d'autre part, en tenant compte mois par mois des variations de température relevées sur le site. Faute d'intéresser tous les pisciculteurs, cette partie peut aider le candidat pisciculteur ou l'expert de l'administration pour instruire un dossier d'implantation d'une nouvelle unité de production.

Le travail présenté ici ne constitue encore qu'une étape expérimentale, largement perfectible. Sur l'ossature de programme déjà en place il sera possible de greffer d'autres calculs et de nombreuses extensions sont à l'étude. A brève échéance, il est prévu d'intégrer dans le logiciel la mortalité habituelle et la dispersion individuelle en lot de tête, lot moyen et lot de queue. Les recherches en cours visent aussi à établir un fichier des résultats conservés sur disquette. A la première utilisation, ce fichier recueillerait les prévisions initiales puis, au fil des mises a jour, l'utilisateur pourrait confronter la prévision à la réalité (pesées réelles) et le programme procèderait, le cas échéant, à la révision des prévisions initiales. Chaque fichier centraliserait ainsi les caractéristiques techniques de chaque lot et un programme supplémentaire pourrait à chaque instant faire un bilan regroupant tous les lots en élevage sur le site exploité. La diversité des extensions suggérées impose un long travail qui ne permet pas encore de fournir ce logiciel. L'état d'avancement de ce travail est annuellement présenté lors de stages de formation ouverts à tous les membres de la profession. Quelques pisciculteurs expérimentent chez eux ce logiciel en cours d'évolution. L'exploitation à plus grande échelle nécessite, en plus des extensions suggérées, la traduction de compatibilité avec d'autres micro-ordinateurs.

Dans une étape ultérieure, il est prévu d'intégrer ce programme dans un logiciel plus complet pour l'ensemble de la gestion d'une entreprise de salmoniculture.

L'idéal serait de créer un logiciel de gestion en intégrant la prévision d'exploitation avec optimisation de la productivité, la gestion technique de chaque production et la gestion commerciale de l'entreprise. Si jusqu'à présent c'est la complexité de la gestion technique qui limitait l'entrée de l'outil informatique en pisciculture, ce premier travail doit encourager l'avancée d'une recherche avec la participation d'abord des pisciculteurs eux-mêmes et des divers spécialistes biologistes et informaticiens.

\section{BIBLIOGRAPHIE}

BENSON B.B., KRAUSE D. Jr, 1980. The concentration and isotopic fractionation of gases dissolved in freshwater in equilibrium with the atmosphere. 1-Oxygene. Limnol. Oceanogr. 25, 662-671

BORGSTROM G., 1961. " Fish as food ", volume 1, Academic Press, New-York, 725 p.

BRETT J.R., 1976. Feeding metabolic rates of young sockeye salmon (Oncorhynchus nerka) in relation to ration level and temperature. Fisheries and Marine Service. Technical report, $n^{\circ} 675$. Environment Canada, pp. 1-43.

CEMAGREF (groupement de Bordeaux, Division aménagements littoraux et aquaculture) 1976 Bases de gestion de l'eau en Salmoniculture intensive. Etude $n^{\circ} 4,82$ pages + annexes.

FAURE A., 1976. Bases de gestion de l'eau en Salmoniculture intensive. La Pisciculture Francaise, 46. $11-54$

GREEN E.J., CARRITT D.E., 1967. New tables for oxygen saturation of sea-water. J. Mar. Res, 25, (2), 140-147.

HOCHACHKA P.W., SOMERO G.N., 1971. Biochemical adaptation to the environment. In "Fish Physiology" (W.S. HOAR and D.J. RANDALL éd.). Vol. 6. Academic Press, New-York, pp. 99-156.

HOSKINGS G.E., KIESER D, NASH F., 1979. Computer management of fish health data. Prog. FishCult., 41. (4), 176-180. 
ITAZAWA Y., 1971. An estimation of the minimum of dissolved oxygen in water required for normal life of fish. Bull. Jap. Soc. Sci. Fish., 37, 4.

LELU J.G., 1976. Gestion d'entreprise piscicole et mise en place d'un tableau de bord. Mémoire de fin d'étude. Ecole Supérieure d'Agriculture de Purpan, Toulouse.

LIAO P.B., (c/O KRAMER, CHIN et MAYO consult. engen.), 1971. Water requirements of salmonids. Prog. Fish-Cult., 33, (4), 210-215.

LOVE R.M., 1980. " Biology of Fish ", Volume 2, Academic Press, New-York. 943 pages.

MORTIMER C.H., 1981. The oxygen content of air satured fresh water over ranges of temperature and atmospheric pressure of limnological interest. Mitt. Internat. Verein. Limnol., 22, 1-23.

PROSSER C.L., BROWN F.A., 1961. "Comparative Animal Physiology ", 2e éd., W.B. Saunders Company, Philadelphie.

SPARRE P., 1976. A markovian decision process applied to optimization of production planning in fish farming. Meddr. Danm. Fisk. Og Havunders N.S., 7, 111-191

SPEECE R.E., 1973. Trout metabolism characteristics and the rational design of nitrification facilities for water reuse in hatcheries. Trans. Am. Fish. Soc., 102, (2), 323-334.

THOMAS S., HUGHES G.M., 1982. A study of the effects of hypoxia on the acid-base status of rainbow trout blood using an extracorporeal blood circulation. Respiration Physiology, 49, $371-382$.

TUFFERY G., 1979. L'utilisation de l'informatique pour la gestion de la santé en pisciculture. Bull. Cent. Et. Rech. Sci., Biarritz, 12, (3), 519-528.

WINBERG G.G., 1960. Rate of metabolism and food requirements of fishes. Bielorussian State University. Minsk. Trans. Ser. Fish. Res. Bd. Can., p. 194. 